\section{Zur Rahmensetzung verpflichtet}

\author{
Bereits der Begriff Integrierte Produktpolitik (IPP) zeigt: Es geht hier auch um Politik. Staatli- \\ ches Handeln ist schon bisher im Bereich Produkte gegenwärtig. Die Ausgestaltung des politi- \\ schen Rahmens im Sinne einer IPP muss jeweils auf die unterschiedlichen Phasen des Lebenszy- \\ klus zugeschnitten sein. Hierzu ist in Abstimmung mit gesellschaftlichen Akteuren eine Konzep- \\ tion auszuarbeiten.
}

$\mathrm{D}$

Von Eckart Meyer-Rutz er Staat ist in eine IPP involviert. Diese Tatsache sagt allerdings noch nichts über die Art und Intensität seines Mitwirkens aus. Um dies zu verdeutlichen, sollte zunächst an die IPPDefinition von Rubik erinnert werden: ,Integrierte Produktpolitik setzt an Produkten und Dienstleistungen und deren ökologischen Eigenschaften entlang des gesamten Lebensweges an; sie zielt auf die Verbesserung ihrer ökologischen Eigenschaften ab und fördert hierzu Innovationen von Produkten und Dienstleistungen“ (1).

Wir erkennen hier, dass der Staat durchaus schon jetzt eine Rolle spielt. Zunächst stand für staatliche Regelungen zwar die Produktionsanlage und die Regelung ihrer Zulassung im Vordergrund. Dann hat sich das Interesse jedoch auf die Produktentsorgung und ihre Regelung erweitert. Die Folgen sind das Kreislaufwirtschaftsgesetz und zahlreiche, auf seiner Grundlage erlassene Verordnungen. Der Staat hat hier also das klassische Mittel des Ordnungsrechts in Anspruch genommen. Auch im Bereich der Produkte und Stoffe hat der Staat mehrfach auf dieses Instrumentarium zurückgegriffen. Dazu ist er schon aus verfassungsrechtlichen Überlegungen heraus verpflichtet, wenn es um Produkte geht, die Leben und Gesundheit des Einzelnen nachteilig beeinflussen können. Die Beispiele sind bekannt; etwa Herstellungs- oder Anwendungsverbote auf Grund des Chemikalienrechts, des Lebensmittel- oder des Arzneimittelrechts. Staatliches Handeln ist hier teilweise und zunehmend auch auf Vorgaben aus dem europäischen Gemeinschaftsrecht zurückzuführen.

Damit ist aber nur ein Aspekt staatlichen Handelns angesprochen. Die weitere Ausdehnung der Umweltpolitik auf das Feld des Produktes in seiner Gesamtheit hat zu einer Vielfalt staatlichen Handelns gefiuhrt, die der Vielfalt von Handlungsfeldern in der IPP entspricht. Das lässt sich entlang des Lebensweges eines Produktes darstellen.
Im Lebensabschnitt Produktinnovation/ Produktdesign geht es um betriebswirtschaftliche und betriebsorganisatorische Abläufe, mithin um einen Kernbereich unternehmerischer Verantwortung. Staatliche Politik sollte nicht versuchen, es hier besser zu wissen als Ingenieure oder Betriebswirte. In diesem Stadium muss es darauf ankommen, ökologische Innovationen attraktiver zu machen. Dies kann in Form gezielter Forschungsförderungspolitik, in Erarbeitung von Informationsangeboten, zum Beispiel in Gestalt von Leitfäden für die Wirtschaft, oder durch Förderung der Nachfrage nach ökologischen Produkten geschehen.

\section{Vielfalt staatlichen Handelns}

Darüber hinaus kann der Staat im Bereich produktgestaltender Normen auch die inhaltliche und organisatorische Einbeziehung von Umweltschutzinteressen unterstuitzen und sich für eine höhere Transparenz der Normungsarbeit einsetzen.

Im Lebensabschnitt Produktnutzung greift Politik traditionell auf die Mittel der Beratung und Aufklärung des einzelnen Produktnutzers zurück. Hier ist die Mitarbeit von Umweltverbänden und Verbraucherverbänden als ,Transmissionsriemen" von großer Bedeutung. Das heißt: Staatliche Politik in Form ordnungsrechtlicher Vorgaben wäre hier, wie im Bereich der Produktinnovation, weitgehend ineffizient.

Politik nutzt hier auch Anreizinstrumente, deren bekanntestes der „Blaue Engel“ als Kennzeichnung besonders umweltfreundlicher Produkte und Dienstleistungen ist. Dies geschieht im Gegensatz zu Kennzeichnungspflichten etwa nach dem Arzneimittelrecht freiwillig. Schließlich kann Politik den Einsatz von Ökobilanzen dadurch fördern, dass sie die Herstellung eines gemeinsamen Grundverständnisses über Struktur und Einsatzbedingungen dieses schwer handhabbaren Instrumentes erreicht und dabei auch das in der internationalen Normung hierzu Erreichte berücksichtigt.
Bei der Vergabe öffentlicher Aufträge bewegt der Staat sich sozusagen auf seinem eigenen Territorium. Daraus ist aber nicht zu schließen, dass er bei der Vergabe ohne Beschränkungen handeln könnte. Staatliche Regelungen - häufig europarechtlichen Ursprungs - geben ihm Kriterien als Beschränkung vor. Dazu gehört auch das Kriterium Umweltschutz.

Im Lebensabschnitt Produktentsorgung ist die Politik dazu aufgefordert, die bisherigen vielfältig ausgeprägten ordnungsrechtlichen aber auch privatrechtlichen Instrumente in einem Gesamtkonzept der übergreifenden Produktverantwortung zusammenzufassen. Dies würde seinerseits wieder positive Rückwirkungen auf Produktentwicklung und Produktnutzung haben.

Damit ist deutlich geworden, dass der Staat in unterschiedlichen Formen und mit unterschiedlichem Regelungsanspruch im Bereich der IPP auftritt. Dabei setzt er vor allem auf die Förderung eines gesteigerten Umweltbewusstseins beim Einzelnen, welches dann auch zur ökologischen Verbesserung von Produkten und Dienstleistungen führen soll.

Grundsätzlich gilt für den Staat auch hier die allgemeine Regel, dass er es ist, der den Handlungsrahmen setzt, der dann durch die gesellschaftlichen Akteure ausgefüllt wird. Zu der Rahmensetzung gehört es auch, eine Konzeption der IPP zu entwickeln. Dies ist nicht nur eine politische „Option“, sondern vielmehr eine politische Verpflichtung, um den zukünftigen Weg der IPP vorzuzeichnen. Diese Konzeption muss mit den gesellschaftlichen Akteuren abgestimmt und auch in Abstimmung mit ihnen verwirklicht werden. Die gemeinsame Verantwortung, die Staat und Gesellschaft für die Umwelt tragen, hat dafür eine tragfähige Grundlage geschaffen.

\section{Anmerkung}

(1) Rubik, Frieder: Innovationen durch die Umweltpolitik Integrierte Produktpolitik in Deutschland. IÖW, Heidelberg/ Berlin 2000, S. 27.

\section{Der Autor}

Eckart Meyer-Rutz ist Ministerialrat im Bundesumweltministerium (BMU). Er leitet seit 1999 das Referat $G$ I 5 Produktbezogener Umweltschutz. Kontakt: BMU, Referat G I 5, Alexanderplatz 6, 11055 Berlin. Tel. 01888/ 305-2260, Fax -3339, E-mail: meyer.eckart@bmu.de 
(c) 20I0 Authors; licensee IÖW and oekom verlag. This is an article distributed under the terms of the Creative Commons Attribution Non-Commercial No Derivates License (http://creativecommons.org/licenses/by-nc-nd/3.o/), which permits unrestricted use, distribution, and reproduction in any medium, provided the original work is properly cited. 